\title{
Orbital radiotherapy plus three-wall orbital decompression in a patient with rare ocular manifestations of thyroid eye disease: case report
}

Shuo Zhang, Yang Wang, Sisi Zhong, Xingtong Liu, Yazhuo Huang, Sijie Fang, Ai Zhuang, Yinwei Li, Jing Sun, Huifang Zhou* and Xianqun Fan ${ }^{*}$

\begin{abstract}
Background: Thyroid eye disease (TED) is a debilitating autoimmune orbital disease that is often a result of Graves' disease. Dysthyroid optic neuropathy (DON) is a rare but sight-threatening manifestation of TED with therapeutic challenges that can potentially lead to visual loss.

Case presentation: A 74-year-old man experienced active TED with extremely severe redness and swelling of the conjunctiva, loss of visual acuity and exacerbation of disfiguring proptosis. Computed tomography revealed the involvement of extraocular muscles resulting in optic nerve compression. He was in poor general condition and was intolerant to steroids. To achieve the optimal operating conditions for orbital decompression surgery, the patient was initially treated with orbital radiotherapy. The patient responded well, with improvements in clinical activity score and visual acuity.

Conclusion: This case demonstrates a rare and severe case of DON with therapeutic challenges. To date, no cases has been reported of a patient with such severe and unusual ocular manifestations. Early awareness of the occurrence of optic nerve compression and prompt treatment are important to prevent irreversible outcomes. Orbital radiotherapy should be considered as a useful surgery-delaying alternative for DON, especially in patients who have contraindications to steroids.
\end{abstract}

Keywords: Thyroid eye disease, Dysthyroid optic neuropathy, Orbital radiotherapy, Three-wall decompression

\section{Background}

Thyroid eye disease (TED) is an autoimmune orbital disease characterized by inflammation and edema of the periorbital connective tissues that results in expansion of the extraocular muscles and fat in the orbit [1]. It typically affects patients with hyperthyroidism due to Graves' disease.

Dysthyroid optic neuropathy (DON) is a rare but potentially sight-threatening complication that occurs in patients with TED. Only $3-5 \%$ of patients with TED suffer from DON [2]. High doses of intravenous

\footnotetext{
* Correspondence: fangzzfang@163.com; fanxq@sh163.net

Department of Ophthalmology, Ninth People's Hospital, Shanghai Jiao Tong University School of Medicine, No. 639 ZhiZaoJu Road, Shanghai 200011, China
}

methylprednisolone (iv-MP) therapy are usually the first-line treatment. Orbital decompression surgery should be performed for patients who fail to adequately respond to iv-MP [3]. The management of patients with DON with contraindications to steroids and urgent surgery remains uncertain. Orbital radiation is administered to patients without DON in most cases [3]. However, limited reports have described the efficacy of orbital radiotherapy and subsequent decompression urgery in DON patients.

Herein, we present a rare and severe case of active DON treated with orbital radiotherapy followed by three-wall orbital decompression in a poor surgical candidate previously intolerant to steroids. To the best of our knowledge, a case of active DON with 
such severe and unusual ocular manifestations has not been previously reported. This case has major clinical significance because it highlights the potential severe presentation of DON and suggests that orbital radiotherapy can be an optimal surgery-delaying treatment for patients who have contraindications to steroids.

\section{Case presentation}

A 74-year-old man presented to the Department of Ophthalmology, Shanghai Ninth People's Hospital, for extremely severe redness and swelling of the conjunctiva, loss of visual acuity and exacerbation of disfiguring proptosis. The patient had a history of Graves' disease and underwent a bilateral subtotal thyroidectomy in 1978. He experienced relapse of Graves' disease three months after surgery and was treated with methimazole with limited response. Due to uncontrolled hyperthyroidism, the patient underwent a left total thyroidectomy in 1985 and radioiodine treatment in 2002. He exhibited proptosis 20 years ago and the proptosis had gradually worsened over the previous years. He underwent iv-MP therapy with doses of $0.84 \mathrm{~g}$ (consecutive daily infusions of $0.12 \mathrm{~g}$ for 3 days and $0.08 \mathrm{~g}$ for 6 days) in 2014 and with doses of $1.8 \mathrm{~g}$ (consecutive daily infusions of $0.48 \mathrm{~g}$ for 3 days, $0.24 \mathrm{~g}$ for 1 day and $0.12 \mathrm{~g}$ for 1 day) twice in 2015 with limited response. The patient also suffered from intolerable side effects of steroids, such as dizziness and headache, had profound muscle weakness and was unable to walk. He also experienced iv-MP-related hepatotoxicity with elevated alanine aminotransferase (ALT) levels. The patient had a history of myocardial infarction, atrial fibrillation and multiple lacunar infarctions. He denied any other comorbid conditions such as hypertension and diabetes mellitus.

An ophthalmic examination showed reduction of best corrected visual acuity to hand movement in the right eye and 0.3 in the left eye, eyelids edema, swelling and hyperemia of the caruncle and plica, redness of the conjunctiva, remarkable chemosis and severe conjunctival prolapse (Fig. 1a and b). He was found to have severe restrictions in ocular movement in all directions with pain on attempted upward and downward gaze and spontaneous retrobulbar pain. He suffered from lagophthalmos and severe corneal exposure with diffuse punctate keratopathy and ulcers. Color vision, pupillary function and optic discs were normal in both eyes. His clinical activity score (CAS) was 6 points. Exophthalmometry showed proptosis of $32 \mathrm{~mm}$ of the right eye and $30 \mathrm{~mm}$ of the left eye. His intraocular pressures were $11 \mathrm{mmHg}$ and $12 \mathrm{mmHg}$ for the right and left eyes, respectively. $\mathrm{He}$ exhibited a free triiodothyronine (FT3) level of $3.43 \mathrm{pg} /$ $\mathrm{mL}$, low free thyroxin (FT4) level of $0.54 \mathrm{ng} / \mathrm{dL}$, low thyroid-stimulating hormone (TSH) level of $0.06 \mu \mathrm{IU} / \mathrm{mL}$, thyroid stimulating hormone receptor antibody (TRAb) level greater than $40.00 \mathrm{IU} / \mathrm{L}$, an extremely high thyroid peroxidase antibody (TPOAb) level of $511.50 \mathrm{IU} / \mathrm{mL}$, an elevated thyroglobulin antibody (TgAb) level of $433.10 \mathrm{IU} /$ $\mathrm{mL}$, high C-reactive protein (CRP) level of $2.06 \mathrm{mg} / \mathrm{dL}$,
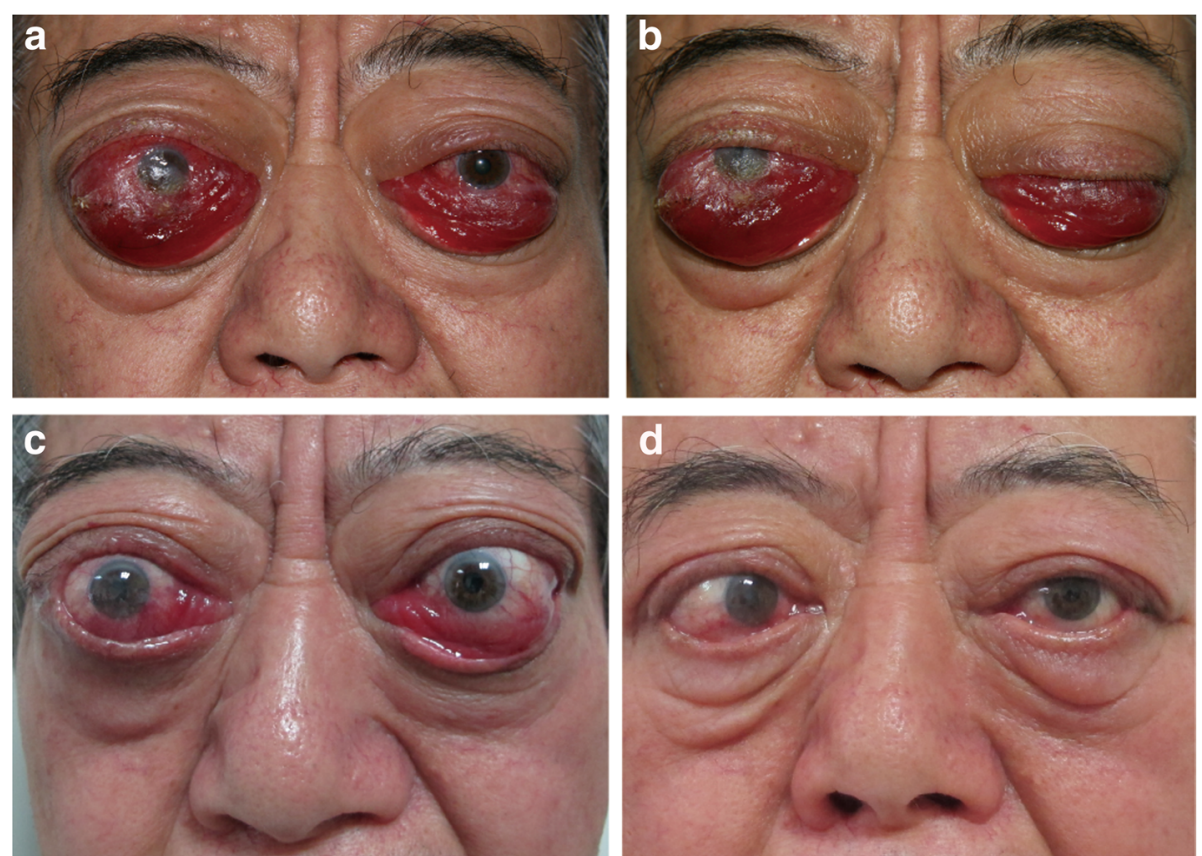

Fig. 1 Ophthalmologic symptoms of the patient. A 74-year-old man developed highly active TED with DON. An ophthalmic examination showed eyelids edema, redness of the conjunctiva, chemosis and severe conjunctival prolapse (a and $\mathbf{b}$ ). One month after orbital radiotherapy (c). Six months after bilateral orbital decompression (d) 
high interleukin-6 (IL-6) level of $127.58 \mathrm{pg} / \mathrm{mL}$, high tumor necrosis factor- $\alpha$ (TNF- $\alpha$ ) level of $259.42 \mathrm{pg} / \mathrm{mL}$, normal immunoglobulin (Ig) G, IgA, IgM and IgG4 levels and an elevated IgE level of $465 \mathrm{IU} / \mathrm{mL}$ (Table 1). The patient received $7.5 \mathrm{mg}$ of methimazole per day and $12.5 \mu \mathrm{g}$ of levothyroxine sodium per day. An orbital computed tomography (CT) scan showed enlargement of the extraocular muscles bilaterally with marked enlargement of the medial rectus and inferior rectus muscles resulting in apical crowding and optic nerve compression (Fig. 2a-c). A diagnosis of active TED with DON and exposure keratitis was made [3].

The patient was evaluated as an urgent case and required prompt intervention (Fig. 3). Bilateral tarsorrhaphy and local therapy were performed to prevent worsening of chemosis and development of corneal ulcers. The patient was suggested to start $500 \mathrm{mg}$ iv-MP for 3 consecutive days, but he had strong concerns about the use of glucocorticoids. At that time, the severe and terrible ocular signs including remarkable chemosis and severe conjunctival prolapse prevented immediate decompression surgery due to high intraorbital pressure and inaccessibility to the small surgical field. Therefore, decompression surgery was deemed high risk for optic nerve impairment. His poor general status due to multiple comorbidities and increased risk under anesthesia also interfered with urgent decompression surgery. Therefore, orbital radiotherapy with 20 Gy per orbit divided into 10 doses was given over a two-week period. Concomitant low-dose oral glucocorticoids

Table 1 Laboratory data before and after radiotherapy

\begin{tabular}{llll}
\hline & $\begin{array}{l}\text { Before } \\
\text { radiotherapy }\end{array}$ & $\begin{array}{l}\text { Two months after } \\
\text { radiotherapy }\end{array}$ & Normal range \\
\hline FT3 & 3.43 & 3.48 & $2.5-3.9 \mathrm{jg} / \mathrm{mL}$ \\
FT4 & 0.54 & 0.45 & $0.58-1.64 \mathrm{ng} / \mathrm{dL}$ \\
TSH & 0.06 & 1.99 & $0.34-5.6 \mathrm{\mu lU} / \mathrm{mL}$ \\
TPOAb & 511.5 & 603.6 & $0-9 \mathrm{IU} / \mathrm{mL}$ \\
TRAb & $>40.00$ & $>40$ & $0-1.75 \mathrm{IU} / \mathrm{L}$ \\
TgAb & 433.1 & 122.9 & $0-115 \mathrm{IU} / \mathrm{mL}$ \\
CRP & 2.06 & 0.60 & $0-0.80 \mathrm{mg} / \mathrm{dL}$ \\
IL-6 & 127.58 & 42.40 & $<3.4 \mathrm{pg} / \mathrm{mL}$ \\
TNF-a & 259.42 & 86.33 & $<8.1 \mathrm{pg} / \mathrm{mL}$ \\
IgG & 9.56 & 11.20 & $7-16 \mathrm{~g} / \mathrm{L}$ \\
IgA & 2.23 & 2.23 & $0.7-4 \mathrm{~g} / \mathrm{L}$ \\
IgM & 0.54 & 0.55 & $0.4-2.3 \mathrm{~g} / \mathrm{L}$ \\
IgE & 465.0 & 312.0 & $0-100 \mathrm{IU} / \mathrm{mL}$ \\
IgG4 & 0.555 & 0.637 & $0.03-2.01 \mathrm{~g} / \mathrm{L}$ \\
\hline I & &
\end{tabular}

Abbreviations: FT3 free triiodothyronine, FT4 free thyroxin, TSH thyroidstimulating hormone, TRAb thyroid stimulating hormone receptor antibody, $T P O A b$ thyroid peroxidase antibody, $\operatorname{TgAb}$ thyroglobulin antibody, $I g$ immunoglobulin, CRP C-reactive protein, IL-6 interleukin-6, TNF- $a$ tumor necrosis factor-a were administered. The chemosis, conjunctival prolapse and corneal lesions significantly improved within 1 month (Fig. 1c). Spontaneous retrobulbar pain and pain on attempted eye movement were also significantly decreased. His CAS was also reduced by 2 points. Thus, an additional 10 Gy per orbit divided into in 5 doses was given. However, the impairment in best corrected visual acuity remained. Orbital radiotherapy significantly improved the ocular signs and offered the possibility of decompression surgery.

Two months later, after extensive discussion of the risks and benefits of surgery, such as haemorrhage, cerebrospinal fluid (CSF) leakage, postoperative diplopia, postoperative lateral orbit depression and scarring, the patient agreed to proceed with right three-wall orbital decompression surgery under general anesthesia. A horizontal skin incision along with the double eyelid fold and lateral to the lateral canthus was made for lateral wall decompression. The deep lateral wall into the trigone of the greater wing of the sphenoid was removed, and the lateral orbital rim was removed en bloc. Transconjunctival and transcaruncular incisions were made for medial and inferior wall decompression. The patient subsequently underwent left three-wall orbital decompression using the same surgical techniques (Fig. 1d).

Following bilateral orbital decompression, the patient's best corrected visual acuity gradually improved. Six months following surgery, the final best corrected visual acuity improved to 0.5 in both the right and left eyes. The patients exhibited improvement in the eyelids edema and swelling and hyperemia of the caruncle and plica. His CAS was reduced to 2 points. Postoperative CT scans demonstrated relief of bilateral crowding in the orbital apex (Fig. 2d-f).

\section{Discussion and conclusion}

DON is a rare and severe complication of TED that can lead to definite visual loss [3]. Compression of the optic nerve at the orbital apex due to extraocular muscles enlargement and inflammatory reaction are the main causes of DON [2]. The diagnosis of DON is made based on the presence of a decreased visual function due to optic neuropathy secondary to TED [3, 4]. The European Group on Graves' Orbitopathy (EUGOGO) reported a prospective case series of 47 patients to identify clinical manifestations of DON [5]. The EUGOGO study found that patients with DON may not have severe proptosis and orbital inflammation. Evidence of optic nerve compression on imaging was one of the most sensitive clinical features, consistent with the findings in our patient. In our patient, the proptosis was $32 \mathrm{~mm}$ in the right eye and $30 \mathrm{~mm}$ in the left eye, which is far greater than the mean proptosis of $22.1 \mathrm{~mm}$ in both eyes 


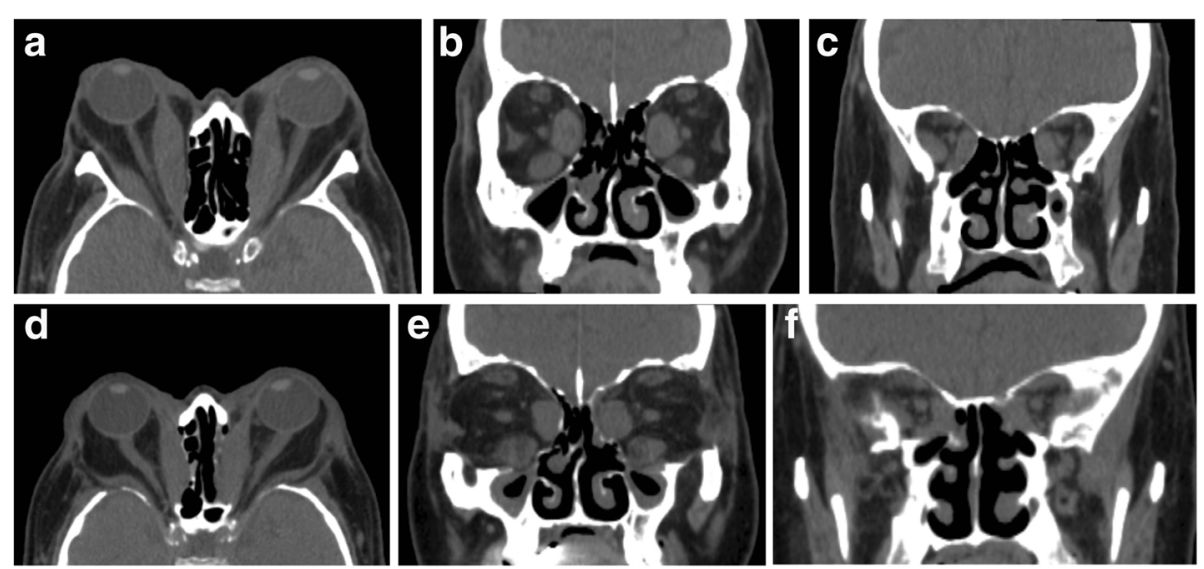

Fig. 2 Preoperative and postoperative computed tomography $(C T)$ of the orbit. Preoperative axial (a) and coronal (b and c) CT images showing proptosis, enlargement of extraocular muscles and apical crowding. Postoperative axial (d) and coronal (e and $\mathbf{f}$ ) CT images showing the reduction in proptosis and relief of apical crowding

reported in the EUGOGO study. To the authors' knowledge, this is the first case of a patient with such severe chemosis and conjunctival prolapse, which made the treatment more perplexing and challenging.

Glucocorticoids, orbital radiotherapy, orbital decompression, immunosuppressive therapy and biological drugs are available for the management of TED [3]. Iv-MP and prompt orbital decompression, if necessary, are is still considered to be the standard treatment for DON [3]. A randomized trial including 15 patients with active TED and DON suggested that immediate surgery does not result in better outcomes, and systemic glucocorticoids appeared to be the optimal first-line treatment [6]. To the best of our knowledge, no reports are available regarding the treatment of patients with DON with contraindications to steroids and surgical intolerance. Orbital radiotherapy plays an important role in controlling the inflammatory process of TED by inducing apoptosis or disrupting the functions of $B$ and $T$ lymphocytes, macrophages, or orbital fibroblasts and therefore reducing the secretion of proinflammatory cytokines from activated lymphocytes [7, 8]. A total dose of 20 Gy is commonly used $[9,10]$. Grassi et al. reported significant early reduction in CAS and ocular motility disturbances after orbital radiotherapy in patients without DON [8]. Another study demonstrated that all patients with TED showed regression of the disease with combined iv-MP and orbital radiotherapy or iv-MP therapy alone. Two of fifty-nine patients undergoing iv-MP therapy developed DON during the follow-up period, but no patients receiving combined iv-MP and orbital radiotherapy developed DON [11]. In addition, some studies have indicated that the high incidence of IgE elevation in Graves' disease suggested a difference in the autoimmune processes of the disease with and without IgE elevation [12, 13]. We found elevated serum IgE levels in our case, suggesting that IgE may also participate in the immunopathogenesis of TED. The patient's IgE level decreased after orbital radiotherapy. The existing literature provides evidence of the efficacy of radiotherapy and its protective role against TED. Therefore, we advocate orbital radiation as the ideal therapy in patients with DON who have contraindications to steroids and cannot tolerate surgery, especially those with elevated IgE levels.

The reported effects of radiotherapy on visual acuity have been variable $[8,14]$. In our case, no significant improvement in visual acuity was found after radiotherapy. Since the recovery of visual acuity is the main goal of treatment in patients with DON, additional orbital decompression is often required. Decompression is the only definitive treatment to relieve apical crowding, save the vision and reduce considerable exophthalmos [3]. Medial and inferior wall decompression led to marked improvement in visual acuity [15]. Balanced medial and lateral wall decompression has shown equal efficacy in terms of saving vision and lowering the rate of postoperative diplopia [16]. Kikkawa et al. proposed graded orbital decompression based on the severity of exophthalmometry, and a mean proptosis reduction of $8.9 \pm 3.4 \mathrm{~mm}$ was obtained in the three-wall decompression group [17]. In the present case, a proptosis reduction of $10 \mathrm{~mm}$ in the right eye and $7 \mathrm{~mm}$ in the left eye was achieved. Orbital radiotherapy ameliorated the inflammatory reactions in the orbit and offered time to prepare for orbital decompression surgery.

Preoperative radiotherapy does not interfere with the outcomes of orbital decompression [18], and may prevent and control relapses in DON [19]. 
A 74-year-old man experienced active TED with extremely severe redness and swelling of the conjunctiva, loss of visual acuity and exacerbation of disfiguring proptosis.

\begin{tabular}{|c|}
\hline Past history \\
\hline $\begin{array}{l}\text { Diagnosis of Graves' disease and bilateral } \\
\text { subtotal thyroidectomy. }\end{array}$ \\
\hline Left total thyroidectomy. \\
\hline Onset of disfiguring proptosis. \\
\hline Radioiodine treatment. \\
\hline $\begin{array}{l}\text { Intravenous methylprednisolone (iv -MP) } \\
1^{\text {st }} \text { time: } 0.12 \mathrm{~g} / \mathrm{d} \text { for } 3 \text { days and } 0.08 \mathrm{~g} / \mathrm{d} \text { for } 6 \\
\text { days, consecutively. } \\
2^{\text {nd }} \text { time: } 0.48 \mathrm{~g} / \mathrm{d} \text { for } 3 \text { days, } 0.24 \mathrm{~g} / \mathrm{d} \text { for } 1 \text { day } \\
\text { and } 0.12 \mathrm{~g} / \mathrm{d} \text { for } 1 \text { day, consecutively. } \\
3^{\text {rd }} \text { time: the same dose as the } 2^{\text {nd }} \text { time. } \\
\text { Limited response to iv }-\mathrm{MP} \text {. }\end{array}$ \\
\hline
\end{tabular}

History of myocardial infarction, atrial fibrillation and multiple lacunar infarctions.
1978

1985

1996

2002

2014

2015

The patient presented to the Department of Ophthalmology, Shanghai Ninth People's Hospital.

2016

Diagnosis of DON. The CAS was 6 points.

Bilateral tarsorrhaphy and local therapy.

Orbital radiotherapy with 20 Gy per orbit divided into 10 doses given over a two-week period and concomitant low-dose oral methylprednisolone (starting dose of $32 \mathrm{mg} / \mathrm{d}$ for 2 weeks and then reduced by $4 \mathrm{mg}$ per week)

The CAS was reduced to 4 points Additional 10 Gy per orbit divided into in 5 doses.

2017 The CAS remained 4 points.

Right three -wall orbital decompression surgen

Left three-wall orbital decompression surgery.

The CAS was reduced to 2 points.

The patient responded well with improvement in the TED clinical activity score. The DON also improved. His best corrected visual acuity improved gradually to 0.5 in both the right and left eyes.

Fig. 3 Timeline of interventions and outcomes

Shams et al. reported that the rate of DON was significantly reduced in patients receiving orbital radiotherapy in addition to corticosteroids in their study [19]. In our case, preoperative radiotherapy offered optimal operating conditions for orbital decompression surgery and eased the surgery.

In conclusion, DON is a rare disease in patients with TED. Apart from the well-known ocular manifestations of TED, severe conjunctival prolapse and apical crowding can occur, resulting in devastating sight loss. We suggest that orbital radiotherapy can be a temporizing treatment for patients with DON in poor general condition and with contraindications to steroids until the patients are well prepared for orbital decompression. Patients with elevated IgE levels are especially likely to benefit from radiotherapy. The efficacy of orbital radiation for DON has not been well investigated and further studies and clinical trials are needed. 


\section{Abbreviations}

CRP: C-reactive protein; DON: Dysthyroid optic neuropathy; FT3: Free triiodothyronine; FT4: Free thyroxin; Ig: Immunoglobulin; IL-6: Interleukin-6; TED: Thyroid eye disease; TgAb: Thyroglobulin antibody; TNF-a: Tumor necrosis factor-a; TPOAb: Thyroid peroxidase antibody; TRAb: Thyroid stimulating hormone receptor antibody; TSH: Thyroid-stimulating hormone

\section{Acknowledgements}

The authors would like to thank all staff involved in the care of the patient presented in this case report.

\section{Funding}

This study was supported in part by the National Natural Science Foundation of China (81170876, 31600971, 81320108010), Shanghai Municipal Education Commission-Gaofeng Clinical Medicine Grant Suppport (20152228), National High Technology Research and Development Program (863 Program) (2015AA020311); Shanghai Municipal Hospital Emerging Frontier Technology Joint Project (SHDC12012107), and Shanghai Science and Technology Commission Research Project (16411950600, 14411968000).

\section{Availability of data and materials}

The datasets presented in the current report are available from the corresponding author on reasonable request.

\section{Authors' contributions}

SZ drafted the manuscript and performed the literature review. YW, SSZ, XL, $\mathrm{YH}, \mathrm{SF}$ and $\mathrm{AZ}$ performed the ophthalmic assessments and participated in data collection. $\mathrm{YL}$, JS and $\mathrm{HZ}$ managed the patient's ophthalmic conditions and performed the decompression surgery. $\mathrm{HZ}$ and XF reviewed and revised the manuscript. All authors read and approved the final manuscript.

\section{Authors' information}

Not applicable.

\section{Ethics approval and consent to participate}

This case report was approved by the Ethics Committee of Shanghai Ninth People's Hospital (No. [2016]-29). The report was in compliance with the tenets of the Declaration of Helsinki for clinical research.

\section{Consent for publication}

Written informed consent was obtained from the patient for publication of this case report and any accompanying images. A copy of the consent is available for review by the editor of this journal.

\section{Competing interests}

The authors declare that they have no competing interests.

\section{Publisher's Note}

Springer Nature remains neutral with regard to jurisdictional claims in published maps and institutional affiliations.

\section{Received: 11 October 2017 Accepted: 23 January 2018}

\section{Published online: 06 February 2018}

\section{References}

1. Bahn RS. Graves' ophthalmopathy. N Engl J Med. 2010;362(8):726-38

2. Bartalena L, Pinchera A, Marcocci C. Management of Graves' ophthalmopathy: reality and perspectives. Endocr Rev. 2000;21(2):168-99.

3. Bartalena L, Baldeschi L, Boboridis K, Eckstein A, Kahaly GJ, Marcocci C, Perros P, Salvi M, Wiersinga WM. European group on graves O: the 2016 European thyroid association/European group on graves' Orbitopathy guidelines for the Management of Graves' Orbitopathy. Eur Thyroid J. 2016; 5(1):9-26.

4. Weis E, Heran MK, Jhamb A, Chan AK, Chiu JP, Hurley MC, Rootman J. Quantitative computed tomographic predictors of compressive optic neuropathy in patients with thyroid orbitopathy: a volumetric analysis. Ophthalmology. 2012;119(10):2174-8.

5. McKeag D, Lane C, Lazarus JH, Baldeschi L, Boboridis K, Dickinson AJ, Hullo Al, Kahaly G, Krassas G, Marcocci C, et al. Clinical features of dysthyroid optic neuropathy: a European group on Graves' Orbitopathy (EUGOGO) survey. Br J Ophthalmol. 2007:91(4):455-8.
6. Wakelkamp IM, Baldeschi L, Saeed P, Mourits MP, Prummel MF, Wiersinga WM. Surgical or medical decompression as a first-line treatment of optic neuropathy in Graves' ophthalmopathy? A randomized controlled trial. Clin Endocrinol. 2005:63(3):323-8.

7. Dolman PJ, Rath S. Orbital radiotherapy for thyroid eye disease. Curr Opin Ophthalmol. 2012;23(5):427-32

8. Grassi P, Strianese D, Piscopo R, Pacelli R, Bonavolonta G. Radiotherapy for the treatment of thyroid eye disease-a prospective comparison: is orbital radiotherapy a suitable alternative to steroids? Ir J Med Sci. 2017;186(3):647-52.

9. Bartalena L, Baldeschi L, Dickinson AJ, Eckstein A, Kendall-Taylor P, Marcocci C, Mourits MP, Perros P, Boboridis K, Boschi A, et al. Consensus statement of the European group on Graves' orbitopathy (EUGOGO) on management of Graves' orbitopathy. Thyroid. 2008;18(3):333-46.

10. Prummel MF, Terwee CB, Gerding MN, Baldeschi L, Mourits MP, Blank L, Dekker FW, Wiersinga WM. A randomized controlled trial of orbital radiotherapy versus sham irradiation in patients with mild Graves' ophthalmopathy. J Clin Endocrinol Metab. 2004;89(1):15-20.

11. Kim JW, Han SH, Son BJ, Rim TH, Keum KC, Yoon JS. Efficacy of combined orbital radiation and systemic steroids in the management of Graves' orbitopathy. Albrecht Von Graefes Arch Klin Exp Ophthalmol. 2016;254(5):991-8.

12. Sato A, Takemura Y, Yamada T, Ohtsuka H, Sakai H, Miyahara Y, Aizawa T, Terao A, Onuma $\mathrm{S}$, Junen $\mathrm{K}$, et al. A possible role of immunoglobulin $\mathrm{E}$ in patients with hyperthyroid Graves' disease. J Clin Endocrinol Metab. 1999; 84(10):3602-5.

13. Komiya I, Yamada T, Sato A, Kouki T, Nishimori T, Takasu N. Remission and recurrence of hyperthyroid Graves' disease during and after methimazole treatment when assessed by IgE and interleukin 13. J Clin Endocrinol Metab. 2001:86(8):3540-4.

14. Hutchison BM, Kyle PM. Long-term visual outcome following orbital decompression for dysthyroid eye disease. Eye. 1995;9(Pt 5):578-81.

15. Liao SL, Chang TC, Lin LL. Transcaruncular orbital decompression: an alternate procedure for graves ophthalmopathy with compressive optic neuropathy. Am J Ophthalmol. 2006:141(5):810-8.

16. Graham SM, Brown CL, Carter KD, Song A, Nerad JA. Medial and lateral orbital wall surgery for balanced decompression in thyroid eye disease. Laryngoscope. 2003;113(7):1206-9.

17. Kikkawa DO, Pornpanich K, Cruz RC Jr, Levi L, Granet DB. Graded orbital decompression based on severity of proptosis. Ophthalmology. 2002;109(7): 1219-24.

18. Baldeschi L, MacAndie K, Koetsier E, Blank LE, Wiersinga WM. The influence of previous orbital irradiation on the outcome of rehabilitative decompression surgery in graves orbitopathy. Am J Ophthalmol. 2008; 145(3):534-40.

19. Shams PN, Ma R, Pickles T, Rootman J, Dolman PJ. Reduced risk of compressive optic neuropathy using orbital radiotherapy in patients with active thyroid eye disease. Am J Ophthalmol. 2014;157(6):1299-305.

\section{Submit your next manuscript to BioMed Central and we will help you at every step:}

- We accept pre-submission inquiries

- Our selector tool helps you to find the most relevant journal

- We provide round the clock customer support

- Convenient online submission

- Thorough peer review

- Inclusion in PubMed and all major indexing services

- Maximum visibility for your research

Submit your manuscript at www.biomedcentral.com/submit
Biomed Central 